\title{
Hyperemesis Gravidarum, Diagnosis, and Pathogenesis
}

\author{
Abanoub Gabra1 ${ }^{*}$, Hanaa Habib ${ }^{2,3}$ and Mariam Gabra ${ }^{4}$ \\ ${ }^{1}$ Department of Obstetrics and Gynecology, Assuit University, Egypt \\ ${ }^{2}$ Florida Dental Hygienist, USA \\ ${ }^{3}$ Assuit General Hospital, Egypt \\ ${ }^{4}$ Registered Nurse Program, Hillsborough Community College, Tampa, Florida, USA
}

*Corresponding author: Abanoub Gabra, Chief Resident, Department of Obstetrics and Gynecology, Assuit University, Egypt, E-mail: abanoub.gabra@med.au.edu.eg

Received date: November 06, 2018; Accepted date: November 30, 2018; Published date: December 05, 2018

Copyright: (c) 2018 Gabra A, et al. This is an open-access article distributed under the terms of the Creative Commons Attribution License, which permits unrestricted use, distribution, and reproduction in any medium, provided the original author and source are credited.

Citation: Gabra A, Habib H, Gabra M (2018) Hyperemesis Gravidarum, Diagnosis, and Pathogenesis. Crit Care Obst Gyne Vol.5 No.1:5.

\section{Abstract}

Nausea and vomiting are common in pregnancy and the condition may be mild or severe disabling disease. The severe form is known as Hyperemesis Gravidarum (HG) which is characterized by dehydration, electrolyte and metabolic imbalances, and nutritional deficiencies that may cause hospital admission. The severity of Nausea and Vomiting in Pregnancy (NVP) may be assessed by Pregnancy Unique Qualification of Emesis (PUQE) which showed a relatively accurate evaluation of the patient's lifestyle including sleeping hours. Focused history and examination are needed to exclude differential diagnosis because the diagnosis of this condition is mainly determined clinically. Moreover, lab investigations are useful tools to assess complications as electrolyte or metabolic imbalances and renal affection. Besides, they help in determine etiologies in refractory cases. Many theories have been hypothesized to contribute to understanding the pathogenesis of HG. They have been postulated based on associations which are either causal, sequential or coincidental. Many efforts are needed to accurately establish these relationships in terms of well-designed studies of adequate sample size. The hormonal theory is one of the main theories involved in the pathogenesis of the disease specifically human Chorionic Gonadotropin (hCG), estrogen, progesterone, and serotonin. Psychological theory has conflicting evidencebased studies. Therefore, more studies are needed to decide whether psychological problems cause $\mathrm{HG}$ or this is a coincidental or sequential relationship. The immunological theory is newly defined and supported by high levels of cellfree fetal DNA and cytokines.

Keywords: Hyperemesis gravidarum; Diagnosis and pathogenesis; Heliobacter pylori

\section{Abbreviations:}

HG: Hyperemesis Gravidarum; NVP: Nausea and Vomiting in Pregnancy; PUQE: Pregnancy Unique Qualification of Emesis;
hCG: human Chorionic Gonadotropin; UTI: Urinary Tract Infection

\section{Introduction}

Nausea and vomiting are common in pregnancy and the condition may be mild or severe disabling disease [1]. The severe form is known as HG which is characterized by dehydration, electrolyte and metabolic imbalances, and nutritional deficiencies that may need hospital admission [1]. According to ACOG, criteria of diagnosis of HG are the exclusion of other causes of vomiting and being complicated by laboratory signs of acute starvation e.g., ketonuria, electrolyte imbalance, acid-base disturbances, and weight loss. Weight loss is usually defined as at least $5 \%$ of pre-pregnancy weight [2]

The International Statistical Classification of Disease and Related Health Problems, Tenth Revision, defines HG as continuous debilitating nausea vomiting before 22 gestational weeks, they further classify it into a mild and severe condition which is complicated by metabolic disturbances such as metabolic alkalosis, dehydration, or electrolyte imbalance such as hypokalemia and hypochloremia $[3,4]$.

In the USA, the prevalence of NVP may reach $90 \%$ which equals millions of women [5]. But HG is a rare condition; its prevalence varies from $0.5 \%-2 \%$ of cases [6]. There are different criteria of diagnosis of HG which make diagnosis varies from one society to another [2]. Regarding ethnicity, it is found to be more often in western and urban populations and is rare among Africans, Native Americans, Eskimos, and most Asian populations. But only a few studies have focused on the racial factors of NVP with conflicting results [5].

NVP and HG are considered the most common cause of hospitalization in the first trimester [6]. This is considered a major financial burden over the health system everywhere, which is not caused only by the cost of drugs and other hospital bills but also by work abstinence [7]. In the UK, mortality from HG was recorded in the $19^{\text {th }}$ century due to the absence of effective management for complications like Wernicke 
encephalopathy and aspiration pneumonia [4]. But with improving treatment, the mortality rate has been dramatically decreased.

\section{Diagnosis}

\section{Assessment of severity}

The severity of NVP may be assessed by PUQE. The PUQE score includes questions about the number of vomiting attacks per day, the length of nausea per day in hours and the number of retching episodes, with a minimum score of 3 and a maximum score of 15 . A score of $<6$ suggests mild HG, 7-12 moderate HG and 13 or more indicates severe HG. The PUQE score may also be used to monitor treatment outcome during hospital admission day by day [8].

Study of Lacasse, et al. compares 12 hours versus 24 hours assessment by PUQE in order to have a more general idea about the severity of condition including the sleeping hours [9]. The 24-hour model was found to be correlated with 12-hour model, but it classified the cases more severely than the 12 hours one. Besides, it was found to be correlated with the deterioration of the quality of life assessed by the SF-12 model [9]. It was also found to be correlated to questionnaires for ability to intake multivitamins daily and visiting the emergency unit for vomiting, higher the PUQE-24 was found to be associated with inability of keeping intake of daily vitamins and higher rate of visits to emergency unit due to vomiting, but sleep pattern was not affected [10]. There are 2 confounding factors that affect the sleep pattern either nauseating pregnant women want to sleep most of the day and night or it may be considered a side effect of antiemetics like antihistamines [10]. As a result of that, the authors summarized that the 24-hour model is valid to assess the severity of NVP in the first trimester [11]

In 2016, RCOG provided physician guidelines for diagnosis and management based on statistics of many studies. They validate the diagnosis of HG based on the triad of more than $5 \%$ prepregnancy weight loss, dehydration, and electrolyte imbalance. NVP should only be diagnosed when onset is in the first trimester of pregnancy and other causes of nausea and vomiting have been excluded. RCOG explained that onset of NVP is in the first trimester and if the initial onset is later, other causes need to be considered and excluded first. Its onset is between the fourth and seventh weeks of gestation, it peaks around the ninth week and recovery occurs by the $20^{\text {th }}$ week in $90 \%$ of patients. They confirmed that the PUQE score can be used to classify the severity of NVP [12].

\section{History}

In patients with NVP, we should consider certain points during history taking which include accurate dating of pregnancy and the onset of symptoms during this pregnancy, past medical history of NVP/HG, using PUQE score to assess severity, hypersalivation may occur in $60 \%$ of cases [5], spitting, loss of weight, inability to keep food and fluids and assessing the deterioration of quality of life. We should also exclude other causes of vomiting which may include: abdominal pain, urinary symptoms, infection, drug history, and chronic Helicobacter pylori infection [12]. It is unlikely for patients to have a fever, chills and rigors, headache and visual disturbance so if these symptoms present, other conditions should be considered [13].

\section{Examination}

The examination should include temperature, pulse, blood pressure, oxygen saturation, respiratory rate, abdominal examination, signs of muscle wasting, dehydration and other examination as guided by history [12]. Dry lips and tongue, decreased skin turgor and reduced urine output are common signs of dehydration which should be excluded as they indicate the need for hospital admission [13]. We should also try to exclude surgical causes like renal colic and appendicitis by examination [13]. Molar pregnancy is suspected by high fundal level not correlated with gestational age and it is confirmed by ultrasound [13].

\section{Investigations}

Investigations are needed to evaluate the disease and determine the severity, they should include urine dipstick for ketones which should be monitored daily till becomes negative for 2 successive days [13]. The mid-stream urine sample should be taken as Urinary Tract Infection (UTI) may be considered as a differential diagnosis. Urea level should be assessed as a severe disease may lead to pre-renal failure due to hypovolemia and decreased renal perfusion, electrolytes panel should be measured to exclude hypokalemia, hyponatremia, metabolic alkalosis and other electrolyte imbalance [12]. Those investigations are very important tests as renal failure and electrolyte imbalances are common [13]. Full blood count should be done as infections like UTI causes leukocytosis, hematocrit value may be raised due to hemoconcentration [12]. Pre-albumin levels may be low which indicate poor food intake especially protein and possible poor fetal weight gain [5].

Random blood glucose may be needed to exclude hypoglycemia as a complication of HG and to exclude diabetic ketoacidosis as a differential diagnosis. An ultrasound scan should be done to exclude molar pregnancy and multiple pregnancies as common obstetrics risk factors, besides determining the viability of intrauterine pregnancy and confirming gestational age [12].

In refractory cases or history of previous admissions, RCOG recommend checking thyroid function tests [12]. TSH levels may be low in HG, due to the structural similarity between TSH and hCG and thyroid hormone levels should return to normal postpartum [5]. The ACOG 2015 guidelines recommend that serum thyroid function studies should be ordered only with signs of hyperthyroidism [2].

Liver function tests should be ordered to exclude liver diseases such as hepatitis or gallstones [5]. Serum calcium, phosphate level, and serum amylase should be assessed to exclude pancreatitis. Arterial blood gases should be done to exclude metabolic disturbances such as metabolic alkalosis and also to monitor the disease [12]. Upper GIT endoscopy can be done safely in pregnancy and can be considered to rule out 
gastritis or peptic ulcer disease; nausea and vomiting were found to be the second most common indication for upper GIT endoscopy in pregnancy after upper GIT hemorrhage [5]. Besides, other investigations such as hemoglobin level, $A B O$ grouping, and Rhesus, VDRL and HIV serology test should be also done as part of routine antenatal care [13].

\section{Differential diagnosis}

Other diagnoses should be excluded as a part of criteria to diagnose NVP [6]. With the help of focused history, examination and investigations, we can exclude other pathological causes of nausea and vomiting include peptic ulcers, cholecystitis, gastroenteritis, hepatitis, pancreatitis, genitourinary conditions such as urinary tract infection or pyelonephritis, metabolic conditions, neurological conditions, and drug-induced nausea and vomiting [12].

The presentation of severe abdominal pain is unexpected to occur with NVP and HG which indicate a further investigation of serum amylase levels and an abdominal sonography or esophageal gastroduodenoscopy [12]. Chronic infection with $H$. pylori can co-exist and serodiagnosis may be needed [14].

\section{Etiology and Theories}

Many etiologies have been theorized to be related to NVP and HG, so we need well-controlled studies of adequate sample size in order to establish the causal relationship between them and the disease [1].

\section{Hormonal theory}

human Chorionic Gonadotropin (hCG): It has been theorized that NVP is related to high hCG production by placenta, as it was discovered in the 1940s by Schoeneck who found that those patients have high level of urinary hCG [1] This association is supported by higher incidence of disease in multiple pregnancies, molar pregnancy, and pregnancy with a fetus affected by Down syndrome [15]. We should be careful in determining the causal relationship because actually, the exact role of hCG is still unclear and in addition to that other conditions with a high level of hCG like choriocarcinoma do not present as vomiting, also some pregnant women with high serum level of hCG do not complain of vomiting [15]. That is why researchers started working on the sensitivity of TSH receptors to hCG and they found that at certain patients, there is a mutation in the extracellular domain of the receptors making them responsive to even normal hCG serum levels [16]. Interestingly it is well known that there is a structural similarity between hCG and TSH [17]. Laboratory bias during measuring hCG is found due to different assays used to detect different serotypes of hCG [15].

Estrogen: High serum level of estrogen in patients with HG points to the role of estrogen hormone in the pathogenesis of the disease [15]. Moreover, nausea and vomiting are well-known side effects of estrogen-containing birth controls [15]. Estrogen decrease intestinal motility and gastric emptying, also it causes fluid shift favors to decrease the acidity of the stomach and potentiate the growth $H$. pylori [15]. Smoking pregnant women have a lower level of estrogen hormone and they are less common to have NVP $[18,19]$.

On the other hand, estrogen level increases with pregnancy but NVP commonly alleviate after the first trimester that is why this causal relation needs to be well studied to allow us figuring out if there are confounding factors or estrogen has an effect modification in presence of other risk factors [5].

Progesterone: Researchers keep in mind the high function of corpus luteum in the first trimester with high progesterone level [20] so that they postulate an association between high progesterone level and NVP [15]. The fact may be attributed to the effect of progesterone on sphincters like the relaxation of lower esophageal sphincter [17]. Level of progesterone goes higher with pregnancy so this association needs further studies like estrogen. Besides, no sufficient data support the correlation between severity of the disease and high level of progesterone [3].

Serotonin: Serotonin has its own role in the pathogenesis of vomiting through its effect on CNS and GIT [15]. Although Borgeat, et al. found that pregnant women with HG do not have high serum levels of serotonin. The role of anti-serotonin drugs in chemotherapy-induced vomiting and their effectiveness in HG are 2 strong evidence-based facts leading to support this association [17].

\section{Psychological theory}

The association between HG and psychological disorders has been already supported by many studies [4] and some studies point to underestimation of this obvious relationship [2]. But the causal relationship between those disorders and HG has not been proved yet $[1,19]$ because studies reported the psychological burden as a consequence of NVP and HG [21]. Moreover, the coincidence of both psychological disease and $\mathrm{HG}$ prolongs the duration of HG [22]. Besides that, a correlation was found between the severity of vomiting and a degree of psychological dysfunction in terms of anxiety, sleep disorder and mood disorder [4].

\section{Immunological theory:}

Cell-free fetal DNA: Studies found that HG patients have a high serum level of fetal cell-free DNA which was also found to be high in patients with preterm labor, preeclampsia and Down's syndrome [23]. Immunological interaction between mother and fetus is a well-balanced condition and essential for the integrity of pregnancy because it regulates invasion of trophoblasts to endometrium [24]. If maternal immunity weakens, trophoblasts would invade the uterus aggressively [25]. This braking mechanism is mediated by the activity of cytotoxic T cells and natural killer cells which was found to be abundant in histopathological examination of the endometrium of patients with HG [23]. Therefore, it has been postulated that abnormal immunological reactions, mediated by cytotoxic $T$ cell and natural killer cells, occur towards trophoblasts of the fetus which causes high serum level of cell-free fetal DNA due to cell induced cytotoxicity [25]. Moreover, correlations were found between 
serum levels of cell-free fetal DNA and both severity of vomiting and hCG level [23].

Cytokines: High serum level of cytokines was found in HG patients especially TNF-alpha which is linked to the secondary messenger process after hormone-receptor interaction [26]. This may be explained by immunological imbalance favoring humoral immunity which is considered one of the physiological changes in pregnancy exacerbated in HG [15]. This overactive humoral immune response increases the level of IL-4 and TNFalpha besides adenosine in order to attenuate this effect [15]. This immune theory is supported by the effectiveness of steroids in some refractory cases [27].

\section{Summary}

Nausea and vomiting are common in pregnancy and the condition may be mild or severe disabling disease. The severe form is known as HG which is characterized by dehydration, electrolyte and metabolic imbalances, and nutritional deficiencies that may cause hospital admission. The severity of NVP may be assessed by PUQE. Focused history and examination are needed to exclude the differential diagnosis. Moreover, lab investigations are useful tools to assess complications. Many theories have been hypothesized to contribute to the pathogenesis of HG. Hormones like estrogen, progesterone, hCG, and serotonin may play a role. Cytokines and fetal cell-free DNA are considered evidence for immunological etiology. A debate is found between researchers regarding psychological etiology because a causal relationship has not been established yet.

\section{References}

1. Philip B (2003) Hyperemesis gravidarum: literature review. WMJ 102: 46-51.

2. London V, Grube S, Sherer DM, Abulafia O (2017) Hyperemesis gravidarum: A review of recent literature. Pharmacology 100: 161-171.

3. Lagiou P, Tamimi R, Mucci LA, Trichopoulos D, Adami HO, et al. (2003) Nausea and vomiting in pregnancy in relation to prolactin, estrogens, and progesterone: a prospective study. Obstet Gynecol 101: 639-644.

4. Ismail SK, Kenny L (2007) Review on hyperemesis gravidarum. Best Pract Res Clin Gastroenterol 21: 755-769.

5. Lee NM, Saha S (2011) Nausea and vomiting of pregnancy. Gastroenterol Clin North Am 40: 309-334.

6. Castillo MJ, Phillippi JC (2015) Hyperemesis gravidarum: a holistic overview and approach to clinical assessment and management. J Perinat Neonatal Nurs 29: 12-22.

7. McCarthy FP, Lutomski JE, Greene RA (2014) Hyperemesis gravidarum: Current perspectives. Int J Womens Health 6 : 719-725.

8. Chhetry M, Thakur A, Uprety DK, Basnet P, Joshi R (2016) Hyperemesis gravidarum in a tertiary care centre in eastern Nepal: A prospective observational study. J Ayub Med Coll Abbottabad 28: 18-21.
9. Lacasse A, Bérard A (2008) Validation of the nausea and vomiting of pregnancy specific health related quality of life questionnaire. Health Qual Life Outcomes 6: 1-6.

10. Ebrahimi N, Maltepe C, Bournissen FG, Koren G (2009) Nausea and vomiting of pregnancy: Using the 24-hour Pregnancy-Unique Quantification of Emesis (PUQE-24) scale. J Obstet Gynaecol Can 31: 803-807.

11. Dochez V, Dimet J, David-Gruselle A, Le Thuaut A, Ducarme G (2016) Validation of specific questionnaires to assess nausea and vomiting of pregnancy in a French population. Int J Gynecol Obstet 134: 294-298.

12. Maltepe C, Koren G (2016) The management of nausea and vomiting of pregnancy and hyperemesis gravidarum-a 2013 update. J Popul Ther Clin Pharmacol 20: 184-192.

13. Loh KY, Sivalingam MFN (2005) Understanding hyperemesis gravidarum. Med J Malaysia 60: 394-399.

14. Golberg D, Szilagyi A, Graves L (2007) Hypermesis grvidarum and H. pylori infection: a systematic review. Obstet Gynecol 110: 695-703.

15. Verberg MF, Gillott DJ, Al-Fardan N, Grudzinskas JG (2005) Hyperemesis gravidarum, a literature review. Hum Reprod Update 11: 527-539.

16. Rodien P, Jordan N, Lefevre A, Royer J, Vasseur C, et al. (2004) Abnormal stimulation of the thyrotrophin receptor during gestation. Hum Reprod Update 10: 95-105.

17. Eliakim R, Abulafia O, Sherer DM (2000) Hyperemesis gravidarum: a current review. Am J Perinatol 17: 207-218.

18. Depue RH, Bernstein L, Ross RK, Judd HL, Henderson BE (1987) Hyperemesis gravidarum in relation to estradiol levels, pregnancy outcome, and other maternal factors: a seroepidemiologic study. Am J Obstet Gynecol 156: 1137-1141.

19. Fell DB, Dodds L, Joseph KS, Allen VM, Butler B (2006) Risk factors for hyperemesis gravidarum requiring hospital admission during pregnancy. Obstet Gynecol 107: 277-284.

20. Yoneyama Y, Suzuki S, Sawa R, Yoneyama K, Doi D, et al. (2002) The T-helper 1/T-helper 2 balance in peripheral blood of women with hyperemesis gravidarum. Am J Obstet Gynecol 187: 1631-1635.

21. McCarthy FP, Khashan AS, North RA, Moss-Morris R, Baker PN, et al. (2011) A prospective cohort study investigating associations between hyperemesis gravidarum and cognitive, behavioural and emotional well-being in pregnancy. PLoS One 6: 1-7.

22. Kjeldgaard HK, Eberhard-Gran M, Benth JS, Vikanes AV (2017) Hyperemesis gravidarum and the risk of emotional distress during and after pregnancy. Arch Women's Ment Health 20: 747-756.

23. Sugito Y, Sekizawa A, Farina A, Yukimoto Y, Saito H, et al. (2003) Relationship between severity of hyperemesis gravidarum and fetal DNA concentration in maternal plasma. Clin Chem 49: 1667-1669.

24. Mullin PM, Bray A, Vu V, Schoenberg-Paik F, MacGibbon K, et al. (2012) No increased risk of psychological/behavioral disorders in siblings of women with hyperemesis gravidarum $(\mathrm{HG})$ unless their mother had HG. J Dev Orig Health Dis 3: 375-379.

25. Sekizawa A, Sugito $Y$, Iwasaki $M$, Watanabe $A$, Jimbo $M$, et al. (2001) Cell-free fetal DNA is increased in plasma of women with hyperemesis gravidarum. Clin Chem 47: 2164-2165.

26. Kaplan PB, Gücer F, Sayin NC, Yüksel M, Yüce MA, et al. (2003) Maternal serum cytokine levels in women with hyperemesis 
gravidarum in the first trimester of pregnancy. Fertil Steril 79: 498-502.
27. Jarvis S, Nelson-Piercy C (2011) Management of nausea and vomiting in pregnancy. BMJ 342: 1-8. 